PAPER

\section{Aerodynamic measurement of a large aircraft model in hypersonic flow}

To cite this article: Bao-Qing Meng et al 2017 Chinese Phys. B 26114702

View the article online for updates and enhancements.

\section{Related content}

Exploring Physics with Computer Animation and PhysGL: Forces and Newton's laws (Part I)

T J Bensky

Semiconductors: Lattice dynamics D K Ferry

Vibro-Acoustic Topology Optimization of Sandwich Panels Partially Treated with MR Fluid and Silicone Rubber Core Layer Masoud Hemmatian and Ramin Sedaghat 


\title{
Aerodynamic measurement of a large aircraft model in hypersonic flow ${ }^{*}$
}

\author{
Bao-Qing Meng(孟宝清) ${ }^{1,2}$, Gui-Lai Han(韩桂来) ${ }^{1, \dagger}$, De-Liang Zhang(张德良) ${ }^{1,2}$, and Zong-Lin Jiang(姜宗林) $)^{1,2}$ \\ ${ }^{1}$ State Key Laboratory of High-temperature Gas Dynamics, Institute of Mechanics, Chinese Academy of Sciences, Beijing 100190, China \\ ${ }^{2}$ University of Chinese Academy of Sciences, Beijing 100049, China
}

(Received 14 April 2017; revised manuscript received 11 June 2017; published online 30 September 2017)

\begin{abstract}
Accurate aerodynamic measurements in the hypersonic flow of large aircraft models in tunnels have practical significance, but pose a significant challenge. Novel aerodynamic force measurement methods have been proposed,but lack theoretical support. The forms of the force signals techniques for signal processing and calculation of aerodynamics are especially problematic. A theoretical study is conducted to investigate the dynamic properties based on models of the draw-rod system and slender rods. The results indicate that the inertia item can be neglected in the rod governing equation; further, the solutions show that the signals of each rod are a combination of aerodynamic signals (with a constant value) and sine signals, which can be verified by experimental shock tunnel results. Signal processing and aerodynamics calculation techniques are also found to be achievable via the flat part of the signals.
\end{abstract}

Keywords: hypersonic, aerodynamic measurement, theoretical study, vibration

PACS: $47.40 . \mathrm{Ki}, 46.40 .-\mathrm{f}, 46.80 .+\mathrm{j}$

DOI: $10.1088 / 1674-1056 / 26 / 11 / 114702$

\section{Introduction}

Accurate aerodynamic measurements in the hypersonic flow of an in-tunnel full-scale aircraft model are important, ${ }^{[1]}$ but are challenging. The measurement of a full-scale aircraft model requires a sufficiently large wind tunnel platform with excellent free stream quality and an innovative support form for the model.

The shock tunnel is an important piece of ground equipment in the aerospace research field. The current tunnel limits the size of the aircraft model, ${ }^{[2,3]}$ but can be used to provide a high total pressure and a high total temperature at relatively low cost. The traditional shock tunnel is only capable of satisfying the measurement of models several hundred millimeters in length, which renders experiments on full-scale models impossible. ${ }^{[4-7]}$ The long-test-duration hypersonic detonationdriven shock tunnel ${ }^{[8]}$ (JF12 shock tunnel) allows full-scale model measurement; the exit diameter of its nozzle is $2.5 \mathrm{~m}$ and the diameter of its test section is $3.5 \mathrm{~m}$. It is capable of reproducing pure air flow with Mach numbers from 5-9 at altitudes of $25-50 \mathrm{~km}$.

An effective support form is also crucial for aerodynamic measurements of a full-scale aircraft model in the shock tunnel. As a typical piece of impulse equipment, the running time of the shock tunnel is very short, generally between approximately 2 and $100 \mathrm{~ms},{ }^{[4,5]}$ which makes the accurate measurement of the aerodynamic force of any model extremely difficult. A running time of several minutes was reported for the conventional tunnel; ${ }^{[9]}$ this duration was found to be far longer than the vibration period of a force measurement struc- ture. Further, the effects of damping were very apparent and the initial vibrations of the force measurement system were decayed. The whole structure can reach the force equilibrium state resulting in quasi-static (and thus highly accurate) measurements. In the shock tunnel, conversely, vibrations, balance, and support of the model are triggered by the impulse force due to the strong primary shock ${ }^{[10-14]}$ during nozzle start-up. The damping effects can be neglected as the order of the magnitude of the test time is identical to that of the force measurement structure period. To this effect, aerodynamic force balance usage is a dynamic measurement process.

Several periods of signals are essential for dynamic signal processing and aerodynamics calculation. Individual signals sensed by the balance form a combination of signals corresponding to the aerodynamic force and vibration. Removal of the vibrational signals represents an essential and critical step processing force signals in the shock tunnel. Several periods (typically more than three) of signals should be tested to ensure accuracy. However, the natural frequencies of a measurement system rely heavily on the support form. More periods of signals would enhance the accuracy of peeled-off vibrational signals. ${ }^{[15,16]}$ Thus, the support form of the model largely determines the overall accuracy of force measurements.

Rear sting supports are the most commonly used support forms in shock tunnels. ${ }^{[17-25]}$ However, the cantilever-beam type limits the frequency, so the mass of the model is limited to a few hundred grams and the length magnitude within the millimeter level. The mass of a full-scale aircraft model is close to $1000 \mathrm{~kg}$. The vibrational frequencies markedly decrease as the

\footnotetext{
*Project supported by the National Natural Science Foundation of China (Grant Nos. 11472281 and 11532014).

${ }^{\dagger}$ Corresponding author. E-mail: hanguilai@imech.ac.cn

(C) 2017 Chinese Physical Society and IOP Publishing Ltd

http://iopscience.iop.org/cpb http://cpb.iphy.ac.cn
} 
model mass increases in these setups. The equation of the frequency for the spring-vibrator model suggests that $\omega \propto M^{-\frac{1}{2}}$, that is the frequency decreases by two orders of the magnitude as the mass varies from 100 grams to $1000 \mathrm{~kg}$. Side support is another commonly used method, ${ }^{[26]}$ which can supply sufficient stiffness in the axial direction, which is typically applied to measure the engine thrust. Again, with increasing model mass (particularly for a full-scale aircraft model with an integrated engine), the side support introduces poor stiffness in both side and normal directions.

Researchers have recently developed a novel aerodynamic force measurement method for large aircraft models in the JF12 shock tunnel. ${ }^{[25]}$ The applicable model mass is about $300-1000 \mathrm{~kg}$ and its length is about $3-5 \mathrm{~m}$. The aircraft model used to explore this method was hung via the draw-rod system (Fig. 1), which includes several slender rods (typically more than six) connected to the model at different positions. Force transducers were linked to both the rods and the rigid base around the wall of the test section. In contrast to the single-surface support form of the rear sting support, the drawrod system provides multipoint support and superior frequency performance.

The preliminary study on this novel aerodynamic measurement method merits some further clarification. The forms of the force signals, for example, are unclear. Effective removal of vibrational signals depends heavily on the signal forms, which are closely related to the overall feasibility and the accuracy of the method. The technique for obtaining the aerodynamics from the force signals should also be further explored. Theoretical support still has these two essential problems. In this study, the theory of static force vectors was investigated in this regard as a series of basic dynamic measurement concepts. Further assessment under the structural dynamics theory was also conducted to investigate the working process of the draw-rod system in the shock tunnel. A few relevant theoretical solutions were proposed, as discussed below.

\section{Superposition of forces and moments}

The superposition of forces and moments is based on the principle of equilibrium within three dimensional force systems i.e., the static theory. It is generally applied in low-speed tunnels with a wire-support system. ${ }^{[27,28]}$ In this study, we also found that it is related to the dynamic aerodynamic measurements.

Before testing the tunnel, the resultant forces and gravity of the model were considered to be in equilibrium as shown in Fig. 1(a) and expressed in Eq. (1). $\boldsymbol{G}$ is the gravity vector and $\boldsymbol{T}_{\mathrm{i}}$ is the inner force vector of each rod. $\boldsymbol{R}_{\mathrm{G}}$ is the position vector from the original point to the acting position of gravity. $\boldsymbol{R}_{\mathrm{i}}$ is the position vector from the original point to the acting position of the inner force of each rod. During testing, the forces on each rod change and the resulting forces, gravity and aerodynamic forces are in equilibrium as shown in Fig. 1(b) and expressed in Eq. (2). $\boldsymbol{D}$ and $\boldsymbol{L}$ are the aerodynamic drag and lift of the aircraft model respectively, $M$ is the aerodynamic moment of the aircraft model during the tunnel test.

$$
\begin{gathered}
\boldsymbol{G}+\sum \boldsymbol{T}_{\mathrm{i}}=0, \\
\boldsymbol{R}_{\mathrm{G}} \times \boldsymbol{G}+\sum \boldsymbol{R}_{\mathrm{i}} \times \boldsymbol{T}_{\mathrm{i}}=0, \\
\boldsymbol{G}+\boldsymbol{L}+\boldsymbol{D}+\sum\left(\boldsymbol{T}_{\mathrm{i}}+\Delta \boldsymbol{T}_{\mathrm{i}}\right)=0, \\
\boldsymbol{R}_{\mathrm{G}} \times \boldsymbol{G}+\boldsymbol{M}+\sum \boldsymbol{R}_{\mathrm{i}} \times\left(\boldsymbol{T}_{\mathrm{i}}+\Delta \boldsymbol{T}_{\mathrm{i}}\right)=0 .
\end{gathered}
$$

Equations (1) and (2) express the aerodynamic forces and moments and combine to form Eq. (3). $\Delta T_{\mathrm{i}}$ is the variation of the force for each rod which can be output by force transducers via a data acquisition instrument $\boldsymbol{r}_{\mathrm{i}}$ is the direction vector of the corresponding rod, which can be extracted via the coordinate system

$$
\begin{aligned}
& \boldsymbol{L}+\boldsymbol{D}=-\sum \Delta \boldsymbol{T}_{\mathrm{i}}=-\sum \Delta T_{\mathrm{i}} \boldsymbol{r}_{\mathrm{i}}, \\
& \boldsymbol{M}=-\sum \boldsymbol{R}_{\mathrm{i}} \times \Delta \boldsymbol{T}_{\mathrm{i}}=-\sum \Delta T_{\mathrm{i}}\left(\boldsymbol{R}_{\mathrm{i}} \times \boldsymbol{r}_{\mathrm{i}}\right) .
\end{aligned}
$$
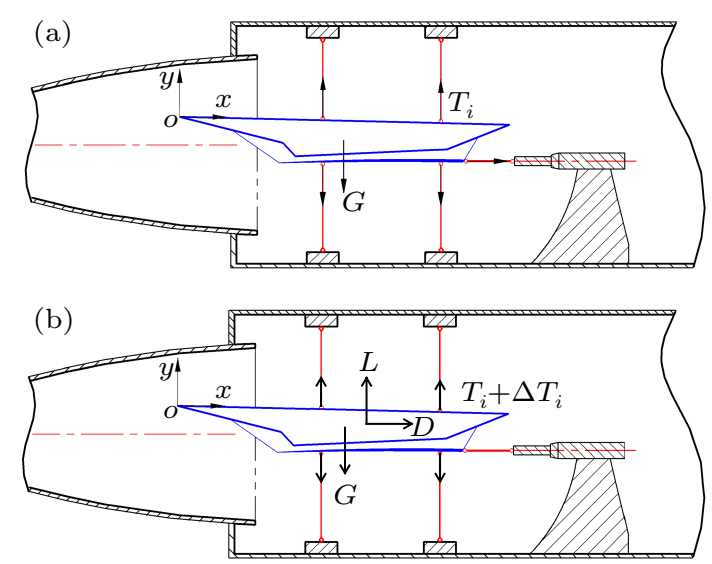

Fig. 1. (color online) (a) Force analysis prior to tunnel testing. (b) Force analysis during testing.

\section{Investigation of dynamic measurement}

The aerodynamic force measurements in the shock tunnel are a dynamic process. Thus, the force measurement theory of the draw-rod system in a shock tunnel is characterized by structural dynamics. The dynamic properties of the aircraft model and rods can be investigated separately. The ultimate objective of applying dynamic theory is to obtain the aerodynamic force with the measured signals of each rod.

The vibration of the whole system can be roughly divided into two stages corresponding to the test process: the forced impulse stage and the steady vibration stage.Signals can be efficiently acquired during steady vibration of the draw-rod system. The following basic assumptions were applied:

i) The aircraft model can be idealized as a rigid body.

ii) The ring support can be considered a rigid base and a motionless part due to its very strong structure and large mass. 
iii) The mass of all rods is below $2 \%$ of the mass of the whole system (including aircraft model, rods and ring support), which can be neglected in forming the mass matrix.

iv) Small deformation assumption and linear elasticity are valid during system vibration.

v) The forces during the steady vibration stage are constant.

vi) The effects of damping can be neglected.

The vibration of the whole system can be simplified as a multidegree-of-freedom structure as describedby ${ }^{[29]}$

$$
[\boldsymbol{M}]\{\ddot{\boldsymbol{y}}\}+[\boldsymbol{K}]\{\boldsymbol{y}\}=\{\boldsymbol{P}(t)\}
$$

$[\boldsymbol{M}]$ is the mass matrix of the system and it is composed of the model mass and inertia moments

$$
[\boldsymbol{M}]=\operatorname{diag}\left[\begin{array}{llllll}
m & m & m & J_{x} & J_{y} & J_{z}
\end{array}\right]
$$

$\{\boldsymbol{y}\}$ is the displacement vector of the aircraft model

$$
\{\boldsymbol{y}\}=\left[\begin{array}{llllll}
u_{x} & u_{y} & u_{z} & \theta_{x} & \theta_{y} & \theta_{z}
\end{array}\right]^{\mathrm{T}},
$$

$[\boldsymbol{K}]$ is the stiffness matrix ${ }^{[30]}$ of the whole system

$$
\begin{aligned}
& {[K]=\sum_{r=1}^{N_{\mathrm{R}}}\left[k_{r}\right]} \\
& {\left[\boldsymbol{k}_{r}\right]_{6 \times 6}=\left[\begin{array}{l}
{[\boldsymbol{E}]_{3 \times 3}} \\
{\left[\boldsymbol{C}_{i}\right]_{3 \times 3}}
\end{array}\right] \frac{E_{r} A_{r}}{L_{r}}\left[\boldsymbol{B}_{r}\right]_{3 \times 3}\left[[\boldsymbol{E}]_{3 \times 3}-\left[\boldsymbol{C}_{r}\right]_{3 \times 3}\right],} \\
& {\left[\boldsymbol{B}_{r}\right]_{3 \times 3}=\left[\begin{array}{lll}
l_{r} l_{r} & l_{r} m_{r} & l_{r} n_{r} \\
m_{r} l_{r} & m_{r} m_{r} & m_{r} n_{r} \\
n_{r} l_{r} & n_{r} m_{r} & n_{r} n_{r}
\end{array}\right] \text {, }} \\
& {\left[\boldsymbol{C}_{r}\right]_{3 \times 3}=\left[\begin{array}{lll}
0 & -z_{r} & y_{r} \\
z_{r} & 0 & -x_{r} \\
-y_{r} & x_{r} & 0
\end{array}\right] \text {. }}
\end{aligned}
$$

$l_{r}, m_{r}, n_{r}$ are the direction cosines of each rod. $x_{r}, y_{r}, z_{r}$ are the coordinates of the connecting positions of each rod. $\left[\boldsymbol{B}_{r}\right]$ is the orientation matrix; it is related solely to the orientations of the rods. $\left[\boldsymbol{C}_{r}\right]$ is the action point matrix, which is comprised solely of the connecting points of each rod and the aircraft model.

\subsection{Theoretical solutions}

The natural frequencies were determined via the properties of the force measurement structure, which can be solved via the following free vibration equation:

$$
[\boldsymbol{M}]\{\ddot{\boldsymbol{y}}\}+[\boldsymbol{K}]\{\boldsymbol{y}\}=\{0\}
$$

Assuming that

$$
\{\boldsymbol{y}\}=\{\boldsymbol{X}\} \sin (\omega t+\phi),
$$

and substituting Eq. (9) into Eq. (8)

$$
\left([\boldsymbol{K}]-\omega^{2}[\boldsymbol{M}]\right)\{\boldsymbol{X}\}=\{0\},
$$

$$
\left|[\boldsymbol{K}]-\omega^{2}[\boldsymbol{M}]\right|=0 .
$$

Let the determinant of the coefficient matrix $\left|[\boldsymbol{K}]-\omega^{2}[\boldsymbol{M}]\right|$ be zero; the eigenvalues $\omega_{i}$ of the matrix are natural frequencies. The characteristic vectors consist of the modal matrix $\{\boldsymbol{X}\}$.

Let $[\boldsymbol{X}]^{\mathrm{T}}$ act on Eq. (4) by left multiplication; then the regular coordinate transformation is applied in Eq. (12)

$$
\{\boldsymbol{y}(t)\}=[\boldsymbol{X}]\{\boldsymbol{\eta}(t)\},
$$

so that the forced vibration equation can be written as follows:

$$
[\boldsymbol{X}]^{\mathrm{T}}[\boldsymbol{M}][\boldsymbol{X}]\{\ddot{\boldsymbol{\eta}}(t)\}+[\boldsymbol{X}]^{\mathrm{T}}[\boldsymbol{K}][\boldsymbol{X}]\{\boldsymbol{\eta}(t)\}=[\boldsymbol{X}]^{\mathrm{T}}\{\boldsymbol{P}(t)\}
$$

The following also holds:

$$
\begin{aligned}
& \left\{\boldsymbol{F}_{p}(t)\right\}=[\boldsymbol{X}]^{\mathrm{T}}\{\boldsymbol{P}(t)\}, \\
& {\left[\boldsymbol{M}_{p}\right]=[\boldsymbol{X}]^{\mathrm{T}}[\boldsymbol{M}][\boldsymbol{X}],} \\
& {\left[\boldsymbol{K}_{p}\right]=[\boldsymbol{X}]^{\mathrm{T}}[\boldsymbol{K}][\boldsymbol{X}],}
\end{aligned}
$$

where $\{\boldsymbol{F}(t)\}$ is the generalized force vector. $\left[\boldsymbol{M}_{p}\right]$ and $\left[\boldsymbol{K}_{p}\right]$ are the generalized mass matrix and the stiffness matrix, respectively. Both $\left[\boldsymbol{M}_{p}\right]$ and $\left[\boldsymbol{K}_{p}\right]$ are diagonal matrices, so equation (13) can be decoupled as follows:

$$
\left[\boldsymbol{M}_{p}\right]\{\ddot{\boldsymbol{\eta}}(t)\}+\left[\boldsymbol{K}_{p}\right]\{\boldsymbol{\eta}(t)\}=\left\{\boldsymbol{F}_{p}(t)\right\} .
$$

The decoupled form of Eq. (15) for generalized displacements can be written as follows:

$$
\begin{aligned}
& \ddot{\eta}_{i}(t)+\omega_{i}^{2} \eta_{i}(t)=\frac{1}{M_{p}(i, i)} F_{p i}(t), \\
& \omega_{i}^{2}=\frac{K_{p(i, i)}}{M_{p(i, i)}} .
\end{aligned}
$$

The solution of Eq. (16) is given by

$$
\eta_{i}(t)=\frac{1}{\mathrm{M}_{p(i, i)} \omega_{i}} \int_{0}^{\tau} F_{i}(\tau) \sin \left[\omega_{i}(t-\tau)\right] \mathrm{d} \tau .
$$

For the actual condition in the shock tunnel, the form of $F_{i}(\tau)$ is a step load with the amplitude denoted as $F_{i}$

$$
\eta_{i}(t)=\frac{F_{i}}{\mathrm{M}_{p(i, i)} \omega_{i}^{2}}\left(1-\cos \omega_{i} t\right) .
$$

The displacement vector of model $\{\boldsymbol{y}(t)\}$ can be solved via Eqs. (12) and (19).

\subsection{Order analysis and rodsignals}

The rods used in the tests are uniform and have equal cross sections. The dynamic process belongs to onedimensional and longitudinal vibration and can be expressed as follows: ${ }^{[29]}$

$$
\rho A \frac{\partial^{2} u}{\partial^{2} t}=E A \frac{\partial^{2} u}{\partial^{2} x}+P_{0} \delta(x-l) .
$$


The symbol $u(x t)$ is the axial displacement and $x$ is the axial direction of the rod. Symbol $t$ denotes the physical time and $A$ is the cross-sectional area of the rod. $E$ is the elasticity modulus of the rod and $P_{0}$ is its exciting force. The term $\rho A \frac{\partial^{2} u}{\partial^{2} t}$ corresponds to the inertia force of the unit length $E A \frac{\partial^{2} u}{\partial^{2} x}$ corresponds to the unit length inertia force, and $P_{0} \delta(x-l)$ is the outer excitation force.

Natural frequencies: ${ }^{[29]}$

$$
\omega_{i}=\frac{(2 i-1) \pi}{2 l} \sqrt{\frac{E}{\rho}}, \quad i=1,2,3, \ldots
$$

Normal modes:

$$
U_{i}(x)=\sqrt{\frac{2}{\rho A l}} \sin \frac{(2 i-1) \pi x}{2 l}, \quad i=1,2,3, \ldots
$$

Generalized force:

$$
q_{i}(t)=\int_{0}^{l} P(x, t) U_{i}(x) \mathrm{d} x .
$$

Normal coordinate:

$$
\eta_{i}(t)=\frac{1}{\omega_{i}} \int_{\mathrm{o}}^{t} q_{i}(\tau) \sin \left[\omega_{i}(t-\tau)\right] \mathrm{d} \tau
$$

Displacement solution:

$$
u(x, t)=\sum_{i=1}^{\infty} \sqrt{\frac{2}{\rho A l}} \sin \left(\frac{(2 i-1) \pi x}{2 l}\right) \eta_{i}(t) .
$$

Based on Eqs. (21a)-(21e), the detailed form of $u(x t)$ is given by

$$
\begin{aligned}
u(x, t)= & \sum_{i=1}^{N} \frac{2}{\rho A l} \frac{P_{0}}{\omega_{i}^{2}} \sin \frac{(2 i-1) \pi}{2} \sin \frac{(2 i-1) \pi x}{2 l} \\
& \times\left[1-\cos \left(\omega_{i} t\right)\right] .
\end{aligned}
$$

The magnitude analysis was conducted for each term in Eq. (20). The orders or the values of each term were evaluated according to the results of the previous tests. Generally, the sum of the first ten modes is sufficient to replace the whole vibration.

$$
\begin{aligned}
& \rho=7850 \mathrm{~kg} / \mathrm{m}^{3}, \\
& d=0.03 \mathrm{~m}, \quad A=\frac{\pi}{4} d^{2}=7.06 \times 10^{-4} \mathrm{~m}^{2}, \\
& l \sim 1 \mathrm{~m}, \\
& f \sim 30 \mathrm{~Hz}, \quad \omega=2 \pi f \sim 189 \mathrm{rad} / \mathrm{s}, \\
& P_{0} \sim 10000 \mathrm{~N}, \\
& N \sim 10, \\
& \sin \frac{(2 i-1) \pi}{2} \sin \left(\frac{(2 i-1) \pi x}{2 l}\right)\left[1-\cos \left(\omega_{i} t\right)\right] \sim 1 .
\end{aligned}
$$

Per Eqs. (22a)-(22e), the displacement of the origin is

$$
u \sim 10^{-7} \mathrm{~m} .
$$

The extension length $\Delta L_{r}$ of each rod is close to the displacement of connecting the position of the rods and aircraft model and is expressed as follows:

$$
\Delta L_{r} \sim u \sim 10^{-7} \mathrm{~m}
$$

The characteristic time equal to the vibrational period is

$$
t_{\mathrm{c}} \sim \frac{1}{f} \sim \frac{1}{30} \mathrm{~s} .
$$

The order of the acceleration is

$$
a_{\mathrm{c}} \sim \frac{\Delta L_{r}}{t_{\mathrm{c}}} \sim 10^{-4} \mathrm{~m} / \mathrm{s}^{2} .
$$

The order of the inertia force is

$$
m a_{\mathrm{c}} \sim 10^{-3} \mathrm{~N}
$$

The ratio of the inertia force and the outer excitation force is

$$
m a_{\mathrm{c}} / P_{0} \sim 10^{-7}
$$

Thus, the inertial force of the rods can be neglected because it is much smaller than the outer excitation forces. Equation (21) can be simplified as follows:

$$
E_{r} A_{r} \frac{\Delta L_{r}}{L_{r}}=P_{0}
$$

The extension lengths can be calculated from the displacement vector $\{\boldsymbol{y}(t)\}$

$$
\Delta L_{r}=\left[-l_{r}-m_{r}-n_{r}\right]\left[\boldsymbol{E}_{3 \times 3}-\boldsymbol{C}_{3 \times 3}\right]\{\boldsymbol{y}(t)\} .
$$

The inner forces of each rod are

$$
N_{r}=\frac{E_{r} A_{r}}{L_{r}}\left[-l_{r}-m_{r}-n_{r}\right]\left[\boldsymbol{E}_{3 \times 3}-\boldsymbol{C}_{3 \times 3}\right][\boldsymbol{X}]_{6 \times 6}\{\boldsymbol{\eta}(t)\}_{6 \times 1},
$$

and the composition of $N_{r}$ is

$$
\begin{aligned}
& N_{r}=\frac{E_{r} A_{r}}{L_{r}}[\boldsymbol{S}]_{1 \times 6}\{\boldsymbol{\eta}(t)\}_{6 \times 1}, \\
& {[\boldsymbol{S}]_{1 \times 6}=\left[-l_{r}-m_{r}-n_{r}\right]\left[\boldsymbol{E}_{3 \times 3}-\boldsymbol{C}_{3 \times 3}\right][\boldsymbol{X}]_{6 \times 6} .}
\end{aligned}
$$

The inner force $N_{r}$ is a combination of a constant value (flat signals) and some sine functions as per the detailed form of $\{\boldsymbol{\eta}(t)\}_{6 \times 1}$ given in Eq. (19), so the form of the signals of each rod has been identified.

\subsection{Methods of dynamic signals processing}

Signal processing is a crucial issue ${ }^{[19-21]}$ inherent to the force measurement in shock tunnels. The signal compositions for each rod in the draw-rod system were determined as described above; the methods of dynamic signals in rod processing were then established to calculate the aerodynamics.

The sinusoidal signals can be removed via signal processing (e.g., the LDSE method). The generalized displacement 
given in Eq. (19) is a constant (independent of time), as indicated by Eq. (33). The relationship between the displacement and the generalized displacement is shown in Eq. (34). It should be noted that $\eta_{i}^{\prime}$ and $\left\{\boldsymbol{y}^{\prime}\right\}$ are independent of $t$.

$$
\begin{aligned}
& \eta_{i}^{\prime}=\frac{F_{i}}{\mathbf{M}_{p}(i, i) \omega_{i}^{2}}=\frac{F_{i}}{K_{p}(i, i)}, \\
& \left\{\boldsymbol{\eta}^{\prime}\right\}=\left[\boldsymbol{K}_{p}\right]^{-1}\{\boldsymbol{F}\}, \\
& \left\{\boldsymbol{y}^{\prime}\right\}=[\boldsymbol{X}]\left\{\boldsymbol{\eta}^{\prime}\right\} .
\end{aligned}
$$

The extension length form of each rod corresponding to the processed signals is similarly given in Eq. (35). The internal force of each rod can be obtained as

$$
\begin{aligned}
& \Delta l_{r} \stackrel{\text { Eq. (30) }}{=}-\left[\begin{array}{lll}
l_{r} & m_{r} & n_{r}
\end{array}\right]\left[\boldsymbol{E}_{3 \times 3}-\boldsymbol{C}_{3 \times 3}\right]\left\{\boldsymbol{y}^{\prime}\right\} \\
& \underline{\underline{\text { Eq. (12) }}}--\left[\begin{array}{lll}
l_{r} & m_{r} & n_{r}
\end{array}\right]\left[\boldsymbol{E}_{3 \times 3}-\boldsymbol{C}_{3 \times 3}\right][\boldsymbol{X}]\left\{\boldsymbol{\eta}^{\prime}\right\} \\
& \stackrel{\underline{\text { Eq. (33b) }}}{\underline{\underline{E^{2}}}}-\left[\begin{array}{lll}
l_{r} & m_{r} & n_{r}
\end{array}\right]\left[\boldsymbol{E}_{3 \times 3}-\boldsymbol{C}_{3 \times 3}\right][\boldsymbol{X}]\left[\boldsymbol{K}_{p}\right]^{-1}\{\boldsymbol{F}\} \\
& \underline{\underline{\text { Eq. (14c) }}}--\left[\begin{array}{lll}
l_{r} & m_{r} & n_{r}
\end{array}\right]\left[\boldsymbol{E}_{3 \times 3}-\boldsymbol{C}_{3 \times 3}\right][\boldsymbol{X}]\left([\boldsymbol{X}]^{-1}[\boldsymbol{K}]^{-1}\right. \\
& \left.\times\left([\boldsymbol{X}]^{\boldsymbol{T}}\right)^{-1}\right)[\boldsymbol{X}]^{\boldsymbol{T}}\{\boldsymbol{P}\} \\
& =\quad-\left[\begin{array}{lll}
l_{r} & m_{r} & n_{r}
\end{array}\right]\left[\boldsymbol{E}_{3 \times 3}-\boldsymbol{C}_{3 \times 3}\right][\boldsymbol{K}]^{-1}\{\boldsymbol{P}\}, \\
& N_{r}=-\frac{E_{r} A_{r}}{L_{r}}\left[\begin{array}{lll}
l_{r} & m_{r} & n_{r}
\end{array}\right]\left[\boldsymbol{E}_{3 \times 3}-\boldsymbol{C}_{3 \times 3}\right][\boldsymbol{K}]^{-1}\{\boldsymbol{P}\} .
\end{aligned}
$$

The resulting force can be written as follows:

$$
\begin{aligned}
& {\left[F_{N}\right]=\left[\begin{array}{llllll}
F_{N x} & F_{N y} & F_{N z} & M_{N x} & M_{N y} & M_{N z}
\end{array}\right]^{\mathrm{T}}} \\
& =\sum_{r=1}^{n R}[[\boldsymbol{E}][\boldsymbol{C}]]^{\mathrm{T}} N_{r}\left[\begin{array}{lll}
l_{r} & m_{r} & n_{r}
\end{array}\right]^{\mathrm{T}} .
\end{aligned}
$$

Further,

$$
\begin{aligned}
& {\left[\boldsymbol{F}_{N}\right]=-\sum_{r=1}^{n R} \frac{E_{r} A_{r}}{L_{r}}[[\boldsymbol{E}][\boldsymbol{C}]]^{\mathrm{T}}\left[\begin{array}{lll}
l_{r} & m_{r} & n_{r}
\end{array}\right]^{\mathrm{T}}\left[\begin{array}{lll}
l_{r} & m_{r} & n_{r}
\end{array}\right]} \\
& \times[[\boldsymbol{E}]-[\boldsymbol{C}]][\boldsymbol{K}]^{-1}\{\boldsymbol{P}\} \\
& \underline{\underline{\text { Eq. (7b) }}}-\sum_{r=1}^{n R}\left[k_{r}\right]\left[\boldsymbol{K}^{-1}\right]^{\mathrm{T}}\{\boldsymbol{P}\} \\
& \stackrel{\text { Eq. (7a) }}{=}-[\boldsymbol{K}][\boldsymbol{K}]^{-1}\{\boldsymbol{P}(t)\} \\
& =-\{\boldsymbol{P}(t)\} \text {. }
\end{aligned}
$$

Thus, the method to obtain the aerodynamics is complete. Each experimental signal contains a flat signal and some sine signals. Numerical methods such as LDSE, as mentioned above, can be applied to extract sine signals as vibrational signals. The frequencies of sine signals are equal to the natural frequencies of the structure. The remaining parts of signals are the flat signals which correspond to a constant force as the inner forces of the rods. The superposition of forces and moments can then be applied, where the constant forces of each rod are similar to the static force $\Delta T_{\mathrm{i}}$ in Eqs. (3a) and (3b). The resultant $\Delta T_{\mathrm{i}}$ value obtained in Eqs. (3a) and (3b) equals the excitation force, i.e., the aerodynamic force.

\subsection{Verification by shock tunnelexperiments}

The signals of the inner forces are a combination of a constant value (flat signals) and some sine signals. Figure 2(a) presents the signals measured by experiments in a shock tunnel. The signals numbered $\mathrm{CH} 9$ and $\mathrm{CH} 10$ represent the inner forces of the rods along the axial direction. The signal compositionswere analyzed by LDSE. ${ }^{[15,16]}$ The compositions of signals CH9 contain flat signals (with an average value of about 1.2) and two sinusoidal signals with frequencies of $23.8 \mathrm{~Hz}$ and $80.2 \mathrm{~Hz}$, as shown in Fig. 2(b). These signal compositions validate the theoretical predictions provided above.
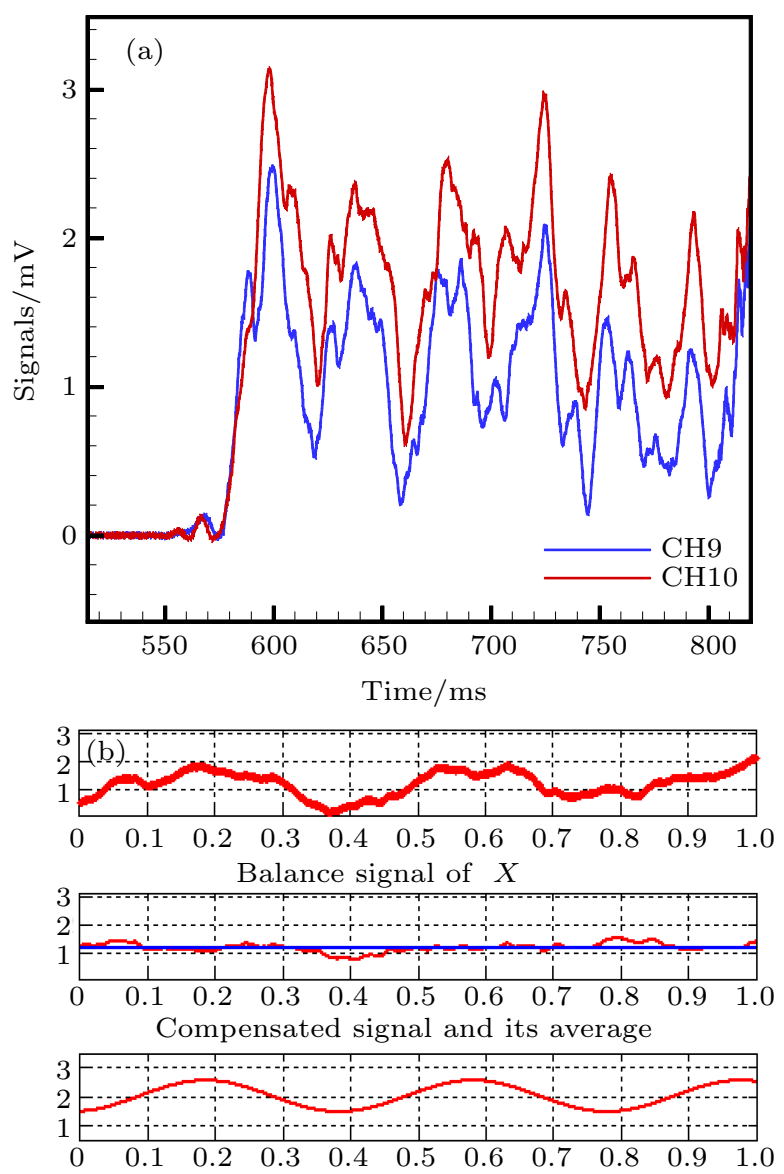
Vibration signal 1, Frequency is: 23.8

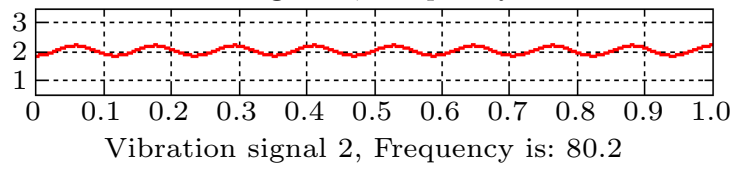

Fig. 2. (color online) (a) Experimental signals of the rods at tail; (b) compositions of $\mathrm{CH} 9$ signals (corresponding to the rod at tail).

The theory that sine signal frequencies equal the natural frequencies of the structure was also validated by experiments. The natural frequencies were measured by knocking experiments. The signals were acquired by accelerometers. The Fourier analysis was applied to measure the natural frequencies. Figure 3 presents the signals of accelerometers along the axial direction with a frequency of $26 \mathrm{~Hz}$. The compositional 
analysis suggests that $23.8 \mathrm{~Hz}$ is the main frequency of the inner force signals, which is very close to $26 \mathrm{~Hz}$. The differences in the frequency values may be due to the signal range selected for LDSE and the position differences of the accelerometers and a force transducer.
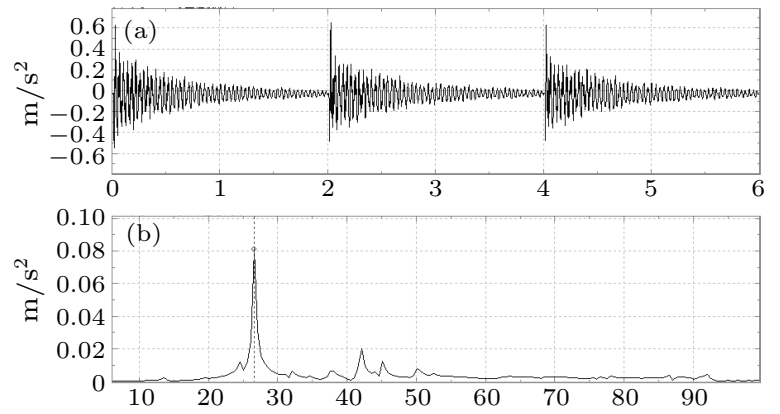

Fig. 3. Signals of accelerometers in the axial directions and Fourier analysis.

\section{Conclusion}

In this study, theoretical support was established for a novel aerodynamic force measurement method applicable to large aircraft models for JF12 shock tunnel tests. The drawrod system and slender rods were modeled and verified numerically.

A physical model was set up for the draw-rod system, and its dynamic properties were analyzed via structural dynamics. A detailed order analysis was also conducted to simplify the vibrational equation of the rods. We found that the inertia force of the rods is much smaller than the outer excitation force. The signals of each rod were identified as a combination of a constant value (flat signal) and sine signals. The experimental results also support this theoretical conclusion.

A method for signal processing and aerodynamics calculation was also proposed here, in which each experimental signal contains a flat signal and some sinusoidal signals. The sine signal frequencies are equal to the natural frequencies of the structure. Vibrational signals can be removed; the remainders are flat signals, which can be converted to a constant force as the inner forces of the rods. The resultant inner constant forces based on the superposition of forces and moments are equal to the aerodynamic forces. These theoretical predictions were validated by experimental tests in an actual shock tunnel.
In the future, we plan to further investigate the interference induced by the rods on aerodynamic measurements.

\section{References}

[1] Wu Z N, Bai C Y, Li J, Chen Z J, Ji S X, Wang D, Wang W B, Xu Y Z and Yao Y 2015 Acta Aeronaut. Astronaut. Sin. 361 (in Chinese)

[2] Wang D P, Zhao Y X, Xia Z X, Wang Q H and Luo Z B 2012 Chin. Phys. Lett. 29084702

[3] Zhang Q H, Yi S H, Zhu Y Z, Chen Z and Wu Y 2013 Chin. Phys. Lett. 30044701

[4] Holden M, KollyJ and Chadwick K 1995 AIAA 33rd Aerospace Sciences Meeting and ExhibitPaper (Reno, NV January 9-12, 1995) 1995-0291

[5] Holden M and Parker R A 2002 Advanced Hypersonic Test Facilities (Reston, Virginia: AIAA Publication) p. 6040

[6] Yu H R, Esser B, Lenartz M and Grönig H 1992 Shock Waves 24

[7] Erdos J, Calleja J and Tamagno J 1994 AIAA 18th AIAA Aerospace Ground Testing ConferencePaper (Colorado Springs, CO June 20-23, 1994) 1994-2524

[8] Jiang Z L and Yu H R 2014 AIAA 52nd Aerospace Sciences Meeting Paper (National Harbor, Maryland 13-17 January 2014) 2014-1012

[9] Buckley M and Sanford D 1998 AIAA 20th AIAA Advanced Measurement and Ground Testing Technology Conference (Albuquerque, NM 15-18 June 1998) 1998-2883

[10] Smith C E 1966 J. Fluid Mech. 244

[11] Saito T andTakayama K 1999 Shock Waves 92

[12] Mouronval A S and Hadjadj A 2005 J. Propul. Power. 212

[13] Mouronval A S, Hadjadj A and Kudryavtsev A N 2003 Shock Waves 125

[14] Wang Y P, Hu Z M and Liu Y F 2016 J. AIAA 544

[15] Luo C T and Yu B 2012 J. Global. Optim. 521

[16] LuoC T, Zhang S LWang C and Jiang Z L 2011 J. Comput. Appl. Math. 2365

[17] Holden MS Wadhams T P MacLean M and Dufrene A 2015 AIAA 20th AIAA International Space Planes and Hypersonic Systems and Technologies Conference (Glasgow, Scotland 6-9 July 2015) 2015-366

[18] Tanno H and Komuro T 2005 T. Jpn. Soc. Aeronaut. S. 48159

[19] Sahoo N, Mahapatra D R, Jagadeesh G, Gopalakrishnan S and Reddy K P J 2007 Measurement 4093

[20] Satheesh K and Jagadeesh G 2009 Measurement 426

[21] Marineau E C 2011 J. Spacecraft. Rockets. 484

[22] Smolinski 2007 AIAA 45th AIAA Aerospace Sciences Meeting and Exhibit (Reno, Nevada 8-11 January 2007) 2007-110

[23] Zhou W J, Ma H D and Bai P 2003 J. Astronaut 246

[24] Trivedi and Menezes V 2012 Measurement 457

[25] Wang Y P, Liu Y F, Yuan C K et al. 2016 Chin. J. Theor. Appl. Mech. 483 (in Chinese)

[26] Hank J, Murphy J and Mutzman R 2008 AIAA 15th AIAA International Space Planes and Hypersonic Systems and Technologies Conference (Dayton, Ohio 28 April-1 May 2008) 2008-2540

[27] Kawamura S, Kino H and Won C 2000 Robotica 181

[28] Xiao Y W, Lin Q, Zheng Y Q and Liang B 2010 Chin. J. Aeronaut. 23 4

[29] Ni Z H Vibration Mechanics (Xi' an: Xi'an Jiao Tong University Press) p. 160

[30] Liu T F, Gao F X and Lü W 2001 Mech. Eng. 231 (in Chinese) 